\title{
Effect of Post Harvest Treatments for Enhancing Shelf Life of 'Litchi'(Litchi chinensis Sonn.)
}

\author{
Ramandeep Kaur, Gurpreet Singh*, Deepika Saxena and Gurpreet Singh
}

Department of Horticulture, Lovely Professional University, Phagwara, Jalandhar, 144411, India

*Corresponding author

\section{Keywords}

Litchi, Shelf life, Chitosan, Ascorbic acid, Citric acid, Oxalic acid

Article Info

Accepted: 08 July 2018 Available Online: 10 August 2018

\section{A B S T R A C T}

A Laboratory experiment was conducted to study the "Effect of Post harvest treatments for enhancing shelf life of 'Litchi' (Litchi chinensis Sonn.)" at the experimental Laboratory, School of Agriculture, Lovely Professional University, Phagwara during summer season of 2017- 18. The experiment was laid out in a Completely Randomized Design (CRD) with three replications. There were eight treatments used in which one control and remaining treatments consist Chitosan (1\%), Ascorbic Acid (5\%), Ascorbic Acid (10\%), Citric Acid (5\%), Citric Acid (10\%), Oxalic Acid (5\%) and Oxalic Acid (10\%). The Fruits were dipped in different solution of these chemicals. The physical and chemical parameters of fruits were collected at $1^{\text {st }}, 3^{\text {rd }}, 5^{\text {th }}$ and $7^{\text {th }}$ day at room temperature. The fruits were spoiled after $7^{\text {th }}$ day at room temperature. Post harvest storage studies revealed that under ambient and cold storage conditions chitosan $1 \%$ treatment showed slower rate of reduction on size of fruits, weight and fruit volume and were significantly affected by treatments. There was significant effect of treatments on chemical parameters like TSS, ascorbic acid, total sugar. The highest TSS was recorded from the fruits treated with oxalic acid 5\%. The maximum total sugar was recorded in Oxalic acid 10\% treatment under both the trails. The Chitosan $1 \%$ treatment will serve as a good alternative for prolonging shelf life. Hence, it can be used as alternative recommendation in the field of post harvest handling of litchi fruits in future.

\section{Introduction}

The litchi (Litchi chinensis Sonn.) is an important subtropical evergreen fruit crop belonging to family Sapindaceae. It is highly specific in climatic requirements and probably due to the reason that its cultivation is restricted to few countries in the world. Worldwide, commercial litchi production is centered largely in the Northern hemisphere, with growing areas including China, Thailand, Taiwan, India, USA and Israel (Menzel,
2001). India is the second largest producer of litchi next to China, with an annual production of 483.30 thousand metric tonnes from an area of 74.40 thousand hectares with productivity of 6.50 metric tons $\mathrm{ha}^{-1}$ In Punjab, commercial cultivation of litchi is restricted in the northern part (Gurdaspur, Pathankot), particularly in the foothills of Himalayas from Tripura to Jammu \& Kashmir and Gangetic plains. Litchi comprises $1.6 \%$ and $1.0 \%$ of the total area and production under fruit crops. Skin browning due to moisture loss is a major limitation in 
the retention of colour in litchi, fruit deteriorating rapidly after harvest, often within 2 to 3 days (Huang and Scott, 1985).

Litchi is very delicate in nature and highly perishable, which accounts for its low shelf life. Wherever, it is grown, its shelf life under ambient conditions is never more than 24-72 hours (Kumar, 2000).

Low temperature storage at $1-5^{\circ} \mathrm{C}$ is used to reduce pathological decay, but has only limited role in reducing pericarp browning. Moreover, the fruits deteriorate rapidly when removed from cold storage. Long-term storage of litchi at low temperature (independently of water loss and browning) is also associated with loss of flavour and hence, poor eating quality (Jiang and Li, 2003). Coating techniques were also carried out to improve litchi fruits storability (Ghosh et al., 1998).

Chitosan, a cationic polysaccharide was used to inhibit pericarp browning of litchi fruits (Zhang and Quantick, 1997). In the recent years, ascorbic acid has been reported for effective control of enzymatic browning of fruits and vegetables (Santerre et al., 1988; Sapers et al., 1989). However, the work done on the effects of ascorbic acid and chitosan coating on pericarp browning and aril decay of litchi fruits is scanty in literature. Therefore, the present study was aimed to investigate the effects of organic acid on pericarp browning and aril decay of litchi fruits. Emphasis was laid on developing the novel strategies of improving -+litchi storability during its post harvest storage.

\section{Materials and Methods}

The experiment was conducted in the Post Graduate Laboratory, Department of Horticulture, School of Agriculture, Lovely Professional University, Phagwara, India. The present investigation entitled "Effect of Postharvest treatments for enhancing shelf life of litchi (Litchi chinensis Sonn)" was carried out soon after harvesting of litchi on 8th June 2017. The experimental material consisted of freshly harvested, mature litchi fruits of cultivars Lal Gola.

The litchi fruits were collected in the bunches, which were harvested from the orchard of Agriculture Department in Gurdaspur. Fruits were kept in muslin cloth bags, covered with a thick layer of green litchi leaves and brought to the laboratory. Jerks during transit were avoided. Fruits were selected according to uniform size and colour, blemished and diseased fruit were discarded.

Prior to the application of post-harvest treatments, destalking of the fruits with the help of sharp scissor was done by retaining only $2 \mathrm{~mm}$ pedicels in each fruit. Pre-cooling is of great importance as it reduces field heat that is necessary for lowering the fast degradative reactions that occurs after harvest. The pre-cooled fruits were air dried prior to the application of treatments. The treated and non-treated fruits were divided into different lots and placed in a perforated brown bag. The experiment was carried out under ambient (25$\left.28^{\circ} \mathrm{C}\right)$ and cold storage $\left(2{ }^{\circ} \mathrm{C}\right)$ conditions.

\section{Results and Discussion}

\section{Length under ambient condition}

The range of fruit length varied from 3.20 to $3.45 \mathrm{~cm}$. The maximum fruit length $3.45 \mathrm{~cm}$ was recorded for $\mathrm{T}_{7}$ (Oxalic Acid 10\%)) followed by $\mathrm{T}_{6}($ Oxalic Acid $5 \%$ ) i.e. $3.36 \mathrm{~cm}$. The minimum fruit length $3.20 \mathrm{~cm}$ was recorded in $\mathrm{T}_{4}$ (Citric Acid 5\%) treatment. As compared to $\mathrm{T}_{4}, \mathrm{~T}_{7}$ showed $17.75 \%$ increase in fruit length. 
Table.1 Effect of organic acids on fruit length $(\mathrm{cm})$ under ambient storage conditions

\begin{tabular}{|l|l|l|l|l|l|}
\hline \multicolumn{2}{|l}{ Fruit Length (cm) } \\
\hline Treatment & Day 1 & Day 3 & Day 5 & Day 7 & Mean \\
\hline $\mathbf{T}_{\mathbf{0}}$ & $3.39^{\mathrm{a}} \pm 0.07$ & $3.26^{\mathrm{a}} \pm 0.01$ & $3.23^{\mathrm{a}} \pm 0.01$ & $3.22^{\mathrm{a}} \pm 0.03$ & 3.30 \\
\hline $\mathbf{T}_{\mathbf{1}}$ & $3.38^{\mathrm{a}} \pm 0.15$ & $3.23^{\mathrm{a}} \pm 0.11$ & $3.20^{\mathrm{ab}} \pm 0.09$ & $3.17^{\mathrm{ab}} \pm 0.098$ & 3.24 \\
\hline $\mathbf{T}_{\mathbf{2}}$ & $3.36^{\mathrm{a}} \pm 0.05$ & $3.22^{\mathrm{a}} \pm 0.06$ & $3.20^{\mathrm{ab}} \pm 0.06$ & $3.18^{\mathrm{ab}} \pm 0.06$ & 3.24 \\
\hline $\mathbf{T}_{\mathbf{3}}$ & $3.4^{\mathrm{a}} \pm 0.12$ & $3.26^{\mathrm{a}} \pm 0.11$ & $3.21^{\mathrm{ab}} \pm 0.01$ & $3.12^{\mathrm{b}} \pm 0.04$ & 3.25 \\
\hline $\mathbf{T}_{\mathbf{4}}$ & $3.36^{\mathrm{ab}} \pm 0.06$ & $3.27^{\mathrm{a}} \pm 0.07$ & $3.13^{\mathrm{b}} \pm 0.07$ & $3.05^{\mathrm{b}} \pm 0.10$ & 3.20 \\
\hline $\mathbf{T}_{\mathbf{5}}$ & $3.36^{\mathrm{a}} \pm 0.09$ & $3.26^{\mathrm{a}} \pm 0.10$ & $3.23^{\mathrm{ab}^{\mathrm{b}} \pm 0.10}$ & $3.17^{\mathrm{ab}} \pm 0.10$ & 3.25 \\
\hline $\mathbf{T}_{\mathbf{6}}$ & $3.39^{\mathrm{ab}} \pm 0.08$ & $3.32^{\mathrm{a}} \pm 0.08$ & $3.29^{\mathrm{ab}} \pm 0.05$ & $3.26^{\mathrm{ab}} \pm 0.04$ & 3.36 \\
\hline $\mathbf{T}_{\mathbf{7}}$ & $3.54^{\mathrm{b}} \pm 0.05$ & $3.35^{\mathrm{b}} \pm 0.05$ & $3.32^{\mathrm{c}} \pm 0.06$ & $3.29^{\mathrm{c}} \pm 0.03$ & 3.45 \\
\hline
\end{tabular}

Width under ambient condition

The range of fruit width varied from 2.88 to $3.22 \mathrm{~cm}$. The maximum value for fruit width was $3.22 \mathrm{~cm}$ recorded for $\mathrm{T}_{2}$ (Ascorbic Acid 5\%) followed by $\mathrm{T}_{3}$
(Ascorbic Acid $10 \%$ ) i.e. $3.19 \mathrm{~cm}$. The minimum value for fruit width was 2.88 $\mathrm{cm}$ recorded $\mathrm{T}_{7}$ (Oxalic Acid 10\%). As compared to $\mathrm{T}_{7}, \mathrm{~T}_{2}$ showed $18.58 \%$ increase in fruit width.

Table.2 Effect of organic acids on fruit width $(\mathrm{cm})$ under ambient storage conditions

\begin{tabular}{|c|c|c|c|c|c|}
\hline \multicolumn{6}{|c|}{ Fruit Width (cm) } \\
\hline Treatment & Day 1 & Day 3 & Day 5 & Day 7 & Mean \\
\hline $\mathbf{T}_{\mathbf{0}}$ & $3.16^{\mathrm{a}} \pm 0.04$ & $3.10^{\mathrm{a}} \pm 0.05$ & $3.07^{\mathrm{a}} \pm 0.06$ & $3.08^{\mathrm{a}} \pm 0.02$ & 3.10 \\
\hline $\mathbf{T}_{\mathbf{1}}$ & $3.15^{\mathrm{ab}} \pm 0.10$ & $3.09^{\mathrm{abc}} \pm 0.12$ & $3.07^{\mathrm{abc}} \pm 0.12$ & $3.13^{\mathrm{a}} \pm 0.14$ & 3.11 \\
\hline $\mathbf{T}_{2}$ & $3.20^{\mathrm{ab}} \pm 0.11$ & $3.25^{\mathrm{ab}} \pm 0.05$ & $3.23^{\mathrm{ab}} \pm 0.05$ & $3.21^{\mathrm{a}} \pm 0.03$ & 3.22 \\
\hline $\mathbf{T}_{3}$ & $3.25^{\mathrm{a}} \pm 0.13$ & $3.20^{\mathrm{abc}} \pm 0.13$ & $3.18^{\mathrm{abc}} \pm 0.13$ & $3.13^{\mathrm{a}} \pm 0.05$ & 3.19 \\
\hline $\mathbf{T}_{4}$ & $3.16^{\mathrm{ab}} \pm 0.13$ & $3.10^{\mathrm{abc}} \pm 0.12$ & $3.08^{\mathrm{abc}} \pm 0.12$ & $3.02^{\mathrm{ab}} \pm 0.04$ & 3.09 \\
\hline$T_{5}$ & $3.04^{\mathrm{ab}} \pm 0.03$ & $3.00^{\mathrm{bc}} \pm 0.05$ & $2.95^{\mathrm{bc}} \pm 0.03$ & $3.10^{\mathrm{a}} \pm 0.10$ & 3.02 \\
\hline$T_{6}$ & $3.09^{\mathrm{a}} \pm 0.09$ & $3.03^{\mathrm{abc}} \pm 0.08$ & $3.01^{\mathrm{abc}} \pm 0.08$ & $3.03^{\mathrm{ab}} \pm 0.06$ & 3.04 \\
\hline $\mathbf{T}_{7}$ & $2.96^{b} \pm 0.07$ & $2.89^{c} \pm 0.07$ & $2.87^{\mathrm{c}} \pm 0.07$ & $2.81^{\mathrm{b}} \pm 0.07$ & 2.88 \\
\hline
\end{tabular}

\section{Weight under ambient condition}

The range of fruit weight varied from 11.55 to $17.86 \mathrm{~g}$. The maximum value for fruit weight was $17.86 \mathrm{~g}$ recorded for $\mathrm{T}_{2}$ (Ascorbic Acid 5\%) followed by $\mathrm{T}_{3}$
(Ascorbic Acid 10\%) i.e. 17.22g. The minimum value for fruit weight was $11.55 \mathrm{~g}$ recorded for $\mathrm{T}_{7}$ (Oxalic Acid $10 \%$ ). As compared to $\mathrm{T}_{7}, \mathrm{~T}_{2}$ showed $54.63 \%$ increase in fruit weight. 
Table.3 Effect of organic acids on fruit weight (g) under ambient storage conditions

\begin{tabular}{|l|l|l|l|l|l|}
\hline \multicolumn{7}{|c|}{ Fruit Weight $(\mathbf{g})$} \\
\hline Treatment & Day 1 & Day 3 & Day 5 & Day 7 & Mean \\
\hline $\mathbf{T}_{\mathbf{0}}$ & $16.60^{\mathrm{b}} \pm 0.69$ & $16.30^{\mathrm{b}} \pm 0.69$ & $16.20^{\mathrm{abc}} \pm 0.70$ & $16.04^{\mathrm{a}} \pm 0.69$ & 16.29 \\
\hline $\mathbf{T}_{\mathbf{1}}$ & $18.50^{\mathrm{ab}} \pm 1.24$ & $17.30^{\mathrm{ab}} \pm 0.60$ & $17.80^{\mathrm{ab}} \pm 1.38$ & $12.80^{\mathrm{ab}} \pm 1.93$ & 16.60 \\
\hline $\mathbf{T}_{\mathbf{2}}$ & $19.70^{\mathrm{a}} \pm 0.92$ & $19.03^{\mathrm{a}} \pm 0.91$ & $18.40^{\mathrm{a}} \pm 0.90$ & $14.05^{\mathrm{ab}} \pm 0.77$ & 17.86 \\
\hline $\mathbf{T}_{\mathbf{3}}$ & $19.50^{\mathrm{a}} \pm 1.10$ & $18.40^{\mathrm{ab}} \pm 1.22$ & $17.70^{\mathrm{ab}} \pm 0.95$ & $13.30^{\mathrm{ab}} \pm 1.18$ & 17.22 \\
\hline $\mathbf{T}_{\mathbf{4}}$ & $18.7^{\mathrm{ab}} \pm 0.79$ & $17.80^{\mathrm{ab}} \pm 1.06$ & $14.90^{\mathrm{bc}} \pm 1.75$ & $9.80^{\mathrm{cd}} \pm 0.62$ & 15.30 \\
\hline $\mathbf{T}_{\mathbf{5}}$ & $19.50^{\mathrm{a}} \pm 0.57$ & $18.20^{\mathrm{ab}} \pm 0.52$ & $14.20^{\mathrm{c}} \pm 0.97$ & $12.50^{\mathrm{bc}} \pm 1.25$ & 16.10 \\
\hline $\mathbf{T}_{\mathbf{6}}$ & $17.60^{\mathrm{ab}} \pm 0.48$ & $16.40^{\mathrm{b}} \pm 0.63$ & $12.90^{\mathrm{cd}} \pm 0.22$ & $11.70^{\mathrm{bcd}} \pm 0.30$ & 14.65 \\
\hline $\mathbf{T}_{\mathbf{7}}$ & $13.80^{\mathrm{c}} \pm 0.65$ & $13.30^{\mathrm{c}} \pm 0.74$ & $10.50^{\mathrm{d}} \pm 0.40$ & $8.61^{\mathrm{d}} \pm 0.56$ & 11.55 \\
\hline
\end{tabular}

\section{Volume under ambient condition}

The range of fruit volume varied from 1.47 to $1.94 \mathrm{cc}$. The maximum value for fruit volume was $1.94 \mathrm{cc}$ recorded for $\mathrm{T}_{4}$ (Citric Acid 5\%) followed by $\mathrm{T}_{3}$
(Ascorbic Acid 10\%) i.e. 1.8cc. The minimum value recorded for fruit volume was $1.47 \mathrm{cc}$ recorded for $\mathrm{T}_{6}$. As compared to $\mathrm{T}_{6}, \mathrm{~T}_{4}$ showed $31.97 \%$ increase in fruit volume.

Table.4 Effect of organic acids on fruit volume (cc) under ambient storage conditions

\begin{tabular}{|l|l|l|l|l|l|}
\hline \multicolumn{5}{c}{ Fruit Volume (cc) } \\
\hline Treatment & Day 1 & Day 3 & Day 5 & Day 7 & Mean \\
\hline $\mathbf{T}_{\mathbf{0}}$ & $1.60^{\mathrm{a}} \pm 0.01$ & $1.65^{\mathrm{a}} \pm 0.008$ & $1.77^{\mathrm{a}} \pm 0.006$ & $1.68^{\mathrm{a}} \pm 0.008$ & 1.63 \\
\hline $\mathbf{T}_{\mathbf{1}}$ & $1.76^{\mathrm{b}} \pm 0.003$ & $1.66^{\mathrm{b}} \pm 0.003$ & $1.58^{\mathrm{b}} \pm 0.003$ & $1.32^{\mathrm{b}} \pm 0.015$ & 1.58 \\
\hline $\mathbf{T}_{\mathbf{2}}$ & $1.74^{\mathrm{c}} \pm 0.003$ & $1.67^{\mathrm{b}} \pm 0.012$ & $1.53^{\mathrm{c}} \pm 0.012$ & $1.33^{\mathrm{bc}} \pm 0.012$ & 1.56 \\
\hline $\mathbf{T}_{\mathbf{3}}$ & $2.15^{\mathrm{a}} \pm 0.008$ & $1.99^{\mathrm{a}} \pm 0.003$ & $1.67^{\mathrm{a}} \pm 0.003$ & $1.45^{\mathrm{a}} \pm 0.003$ & 1.81 \\
\hline $\mathbf{T}_{\mathbf{4}}$ & $1.85^{\mathrm{bc}} \pm 0.005$ & $1.89^{\mathrm{b}} \pm 0.015$ & $1.95^{\mathrm{d}} \pm 0.003$ & $1.99^{\mathrm{a}} \pm 0.014$ & 1.94 \\
\hline $\mathbf{T}_{\mathbf{5}}$ & $1.68^{\mathrm{e}} \pm 0.004$ & $1.64^{\mathrm{b}} \pm 0.015$ & $1.43^{\mathrm{d}} \pm 0.005$ & $1.32^{\mathrm{ab}} \pm 0.026$ & 1.51 \\
\hline $\mathbf{T}_{\mathbf{6}}$ & $1.51^{\mathrm{d}} \pm 0.003$ & $1.67^{\mathrm{b}} \pm 0.008$ & $1.35^{\mathrm{e}} \pm 0.005$ & $1.15^{\mathrm{a}} \pm 0.003$ & 1.47 \\
\hline $\mathbf{T}_{\mathbf{7}}$ & $1.72^{\mathrm{d}} \pm 0.003$ & $1.68^{\mathrm{b}} \pm 0.026$ & $1.55^{\mathrm{bc}} \pm 0.031$ & $1.01^{\mathrm{c}} \pm 2.92$ & 1.54 \\
\hline
\end{tabular}

TSS under ambient condition

The range of TSS varied from to 18.76 to $20.73 \mathrm{Brix}^{\circ}$. The maximum value for TSS was $20.73 \mathrm{Brix}^{\circ}$ recoded for $\mathrm{T}_{2}$ (Ascorbic acid 5\%) followed by $\mathrm{T}_{6}$
(Oxalic Acid 5\%) i.e.20.53Brix ${ }^{\circ}$. The minimum value for TSS was 18.76 Brix $^{\circ}$ recorded for $\mathrm{T}_{3}$ (Ascorbic acid 10\%). As compared to $\mathrm{T}_{3}, \mathrm{~T}_{2}$ showed $10.5 \%$ increase in TSS. 
Table.5 Effect of organic acids on TSS $\left(\mathrm{Brix}^{\circ}\right)$ under ambient storage conditions

\begin{tabular}{|l|l|l|l|l|l|}
\hline \multicolumn{5}{c|}{ TSS } \\
\hline Treatment & Day 1 & Day 3 & Day 5 & Day 7 & Mean \\
\hline $\mathbf{T}_{\mathbf{0}}$ & $20.42^{\mathrm{b}} \pm 0.012$ & $20.68^{\mathrm{a}} \pm 0.06$ & $20.80^{\mathrm{b}} \pm 0.02$ & $20.16^{\mathrm{a}} \pm 0.04$ & 20.51 \\
\hline $\mathbf{T}_{\mathbf{1}}$ & $19.52^{\mathrm{c}} \pm 0.012$ & $19.65^{\mathrm{c}} \pm 0.02$ & $19.79^{\mathrm{c}} \pm 0.02$ & $20.06^{\mathrm{b}} \pm 0.05$ & 19.75 \\
\hline $\mathbf{T}_{\mathbf{2}}$ & $20.45^{\mathrm{b}} \pm 0.05$ & $20.58^{\mathrm{b}} \pm 0.08$ & $20.77^{\mathrm{b}} \pm 0.04$ & $21.12^{\mathrm{a}} \pm 0.05$ & 20.73 \\
\hline $\mathbf{T}_{\mathbf{3}}$ & $18.35^{\mathrm{f}} \pm 0.02$ & $18.65^{\mathrm{f}} \pm 0.03$ & $18.73^{\mathrm{e}} \pm 0.06$ & $19.31^{\mathrm{d}} \pm 0.20$ & 18.76 \\
\hline $\mathbf{T}_{\mathbf{4}}$ & $18.75^{\mathrm{e}} \pm 0.023$ & $19.13^{\mathrm{e}} \pm 0.06$ & $19.20^{\mathrm{d}} \pm 0.02$ & $20.20^{\mathrm{b}} \pm 0.10$ & 19.32 \\
\hline $\mathbf{T}_{\mathbf{5}}$ & $19.25^{\mathrm{d}} \pm 0.023$ & $19.35^{\mathrm{d}} \pm 0.02$ & $19.60^{\mathrm{c}} \pm 0.02$ & $21.20^{\mathrm{a}} \pm 0.02$ & 19.85 \\
\hline $\mathbf{T}_{\mathbf{6}}$ & $20.58^{\mathrm{a}} \pm 0.008$ & $20.77^{\mathrm{a}} \pm 0.03$ & $21.13^{\mathrm{a}} \pm 0.06$ & $19.66^{\mathrm{c}} \pm 0.04$ & 20.53 \\
\hline $\mathbf{T}_{\mathbf{7}}$ & $18.92^{\mathrm{d}} \pm 0.014$ & $19.24 \mathrm{~d}^{\mathrm{e}} \pm 0.03$ & $19.40^{\mathrm{e}} \pm 0.03$ & $20.17^{\mathrm{b}} \pm 0.01$ & 19.43 \\
\hline
\end{tabular}

Acidity under ambient condition

The range of titratable acidity varied from 0.38 to $0.59 \%$. The maximum value for titratable acidity was $0.59 \%$ recorded for $\mathrm{T}_{6}$ (Oxalic Acid 5\%) followed by $\mathrm{T}_{1}$ (Chitosan 1\%) i.e. $0.57 \%$. The minimum value for titratable acidity was $0.38 \%$ recorded for $\mathrm{T}_{0}$ (Control). As compared to $\mathrm{T}_{0}, \mathrm{~T}_{6}$ showed $55.26 \%$ increase in titratable acidity.

Table.6 Effect of organic acids on titratable acidity (\%) under ambient storage conditions

\begin{tabular}{|l|l|l|l|l|l|}
\hline \multicolumn{5}{|c|}{ Acidity (\%) } \\
\hline Treatment & Day 1 & Day 3 & Day 5 & Day7 & Mean \\
\hline $\mathbf{T}_{\mathbf{0}}$ & $0.43^{\mathrm{c}} \pm 0.005$ & $0.42^{\mathrm{d}} \pm 0.017$ & $0.34^{\mathrm{d}} \pm 0.017$ & $0.35^{\mathrm{c}} \pm 0.017$ & 0.38 \\
\hline $\mathbf{T}_{\mathbf{1}}$ & $0.63^{\mathrm{a}} \pm 0.011$ & $0.63^{\mathrm{b}} \pm 0.011$ & $0.56^{\mathrm{b}} \pm 0.008$ & $0.46^{\mathrm{a}} \pm 0.020$ & 0.57 \\
\hline $\mathbf{T}_{\mathbf{2}}$ & $0.55^{\mathrm{b}} \pm 0.005$ & $0.45^{\mathrm{d}} \pm 0.015$ & $0.44^{\mathrm{c}} \pm 0.007$ & $0.38^{\mathrm{bc}} \pm 0.003$ & 0.45 \\
\hline $\mathbf{T}_{\mathbf{3}}$ & $0.64^{\mathrm{b}} \pm 0.014$ & $0.47^{\mathrm{d}} \pm 0.015$ & $0.44^{\mathrm{c}} \pm 0.003$ & $0.44^{\mathrm{a}} \pm 0.015$ & 0.49 \\
\hline $\mathbf{T}_{\mathbf{4}}$ & $0.55^{\mathrm{b}} \pm 0.017$ & $0.46^{\mathrm{d}} \pm 0.017$ & $0.43^{\mathrm{c}} \pm 0.018$ & $0.43^{\mathrm{ab}} \pm 0.011$ & 0.46 \\
\hline $\mathbf{T}_{\mathbf{5}}$ & $0.54^{\mathrm{b}} \pm 0.020$ & $0.53^{\mathrm{c}} \pm 0.020$ & $0.63^{\mathrm{a}} \pm 0.017$ & $0.44^{\mathrm{ab}} \pm 0.015$ & 0.53 \\
\hline $\mathbf{T}_{\mathbf{6}}$ & $0.65^{\mathrm{a}} \pm 0.018$ & $0.64^{\mathrm{ab}} \pm 0.011$ & $0.65^{\mathrm{a}} \pm 0.017$ & $0.43^{\mathrm{ab}} \pm 0.011$ & 0.59 \\
\hline $\mathbf{T}_{\mathbf{7}}$ & $0.65^{\mathrm{a}} \pm 0.021$ & $0.67^{\mathrm{a}} \pm 0.011$ & $0.46^{\mathrm{c}} \pm 0.008$ & $0.45^{\mathrm{a}} \pm 0.015$ & 0.55 \\
\hline
\end{tabular}

Ascorbic acid under ambient condition

The range of ascorbic acid varied from 23.15 to $30.31 \mathrm{mg} / 100 \mathrm{~g}$. The maximum value for ascorbic acid was $30.31 \mathrm{mg} / 100 \mathrm{~g}$ recorded for $\mathrm{T}_{2}$ (Ascorbic
Acid 5\%) followed by $\mathrm{T}_{6}$ (Oxalic Acid $5 \%$ ) i.e. $29.11 \mathrm{mg} / 100 \mathrm{~g}$. The minimum value for ascorbic acid was recorded $23.15 \mathrm{mg} / 100 \mathrm{~g}$ for $\mathrm{T}_{3}$ (Ascorbic Acid $10 \%)$. As compared to $\mathrm{T}_{3}, \mathrm{~T}_{2}$ showed $30.93 \%$ increase in ascorbic acid. 
Table.7 Effect of organic acids on ascorbic acid (mg/100g) under ambient storage conditions

\begin{tabular}{|l|l|l|l|l|l|}
\hline \multicolumn{5}{|c|}{ Ascorbic Acid (mg/100g) } \\
\hline Treatment & Day 1 & Day 3 & Day 5 & Day 7 & Mean \\
\hline $\mathbf{T}_{\mathbf{0}}$ & $29.09 \pm 0.026$ & $28.18^{\mathrm{c}} \pm 0.037$ & $26.73^{\mathrm{c}} \pm 0.026$ & $23.32^{\mathrm{d}} \pm 0.024$ & 26.83 \\
\hline $\mathbf{T}_{\mathbf{1}}$ & $29.32^{\mathrm{b}} \pm 0.016$ & $27.42^{\mathrm{d}} \pm 0.018$ & $25.84^{\mathrm{e}} \pm 0.020$ & $23.92^{\mathrm{c}} \pm 0.039$ & 26.62 \\
\hline $\mathbf{T}_{\mathbf{2}}$ & $31.20^{\mathrm{ab}} \pm 3.34$ & $32.62^{\mathrm{a}} \pm 0.017$ & $30.11^{\mathrm{a}} \pm 0.014$ & $27.33^{\mathrm{a}} \pm 0.029$ & 30.31 \\
\hline $\mathbf{T}_{\mathbf{3}}$ & $25.25^{\mathrm{b}} \pm 0.020$ & $23.74^{\mathrm{e}} \pm 0.017$ & $22.73^{\mathrm{e}} \pm 0.026$ & $20.91^{\mathrm{e}} \pm 0.026$ & 23.15 \\
\hline $\mathbf{T}_{\mathbf{4}}$ & $30.14^{\mathrm{a}} \pm 0.034$ & $28.09^{\mathrm{c}} \pm 0.045$ & $26.40^{\mathrm{d}} \pm 0.051$ & $22.32^{\mathrm{d}} \pm 0.033$ & 26.73 \\
\hline $\mathbf{T}_{\mathbf{5}}$ & $31.36^{\mathrm{b}} \pm 0.023$ & $29.68^{\mathrm{b}} \pm 0.020$ & $26.83^{\mathrm{c}} \pm 0.027$ & $24.22^{\mathrm{c}} \pm 0.023$ & 28.02 \\
\hline $\mathbf{T}_{\mathbf{6}}$ & $31.69^{\mathrm{a}} \pm 0.026$ & $30.40^{\mathrm{b}} \pm 0.175$ & $28.69^{\mathrm{b}} \pm 0.029$ & $25.69^{\mathrm{b}} \pm 0.012$ & 29.11 \\
\hline $\mathbf{T}_{\mathbf{7}}$ & $29.15^{\mathrm{a}} \pm 0.028$ & $26.85^{\mathrm{d}} \pm 0.017$ & $26.63^{\mathrm{c}} \pm 0.030$ & $22.45^{\mathrm{d}} \pm 0.020$ & 26.27 \\
\hline
\end{tabular}

Reducing sugar under ambient condition

The range of reducing sugar varied from 9.80 to $10.40 \%$. The maximum value for reducing sugar was $10.40 \%$ recorded for
$\mathrm{T}_{5}$ (Citric Acid 10\%) followed by $\mathrm{T}_{6}$ (Oxalic Acid 5\%) i.e. 10.32\%. The minimum value for reducing sugar was $9.80 \%$ recorded for $\mathrm{T}_{0}$ (Control). As compared to $\mathrm{T}_{0}, \mathrm{~T}_{5}$ showed $6.12 \%$ increase in reducing sugar.

Table.7 Effect of organic acids onreducing sugar (\%) under ambient storage conditions

\begin{tabular}{|l|l|l|l|l|l|}
\hline \multicolumn{7}{|c|}{ Reducing Sugar (\%) } \\
\hline Treatment & Day 1 & Day 3 & Day 5 & Day 7 & Mean \\
\hline $\mathbf{T}_{\mathbf{0}}$ & $9.75^{\mathrm{e}} \pm 0.005$ & $9.78^{\mathrm{e}} \pm 0.020$ & $9.83^{\mathrm{e}} \pm 0.047$ & $9.85^{\mathrm{d}} \pm 0.026$ & 9.80 \\
\hline $\mathbf{T}_{\mathbf{1}}$ & $10.07^{\mathrm{cd}} \pm 0.011$ & $10.09^{\mathrm{cd}} \pm 0.012$ & $10.15^{\mathrm{cd}} \pm 0.020$ & $10.12^{\mathrm{c}} \pm 0.017$ & 10.10 \\
\hline $\mathbf{T}_{\mathbf{2}}$ & $10.03^{\mathrm{d}} \pm 0.018$ & $10.07^{\mathrm{cd}} \pm 0.020$ & $10.08^{\mathrm{d}} \pm 0.020$ & $10.16^{\mathrm{bc}} \pm 0.029$ & 10.08 \\
\hline $\mathbf{T}_{\mathbf{3}}$ & $10.12^{\mathrm{c}} \pm 0.012$ & $10.17^{\mathrm{c}} \pm 0.014$ & $10.21^{\mathrm{c}} \pm 0.020$ & $10.22^{\mathrm{b}} \pm 0.015$ & 10.18 \\
\hline $\mathbf{T}_{\mathbf{4}}$ & $10.04^{\mathrm{d}} \pm 0.011$ & $10.07^{\mathrm{d}} \pm 0.012$ & $10.17^{\mathrm{c}} \pm 0.017$ & $10.19^{\mathrm{bc}} \pm 0.014$ & 10.11 \\
\hline $\mathbf{T}_{\mathbf{5}}$ & $10.31^{\mathrm{a}} \pm 0.034$ & $10.40^{\mathrm{a}} \pm 0.014$ & $10.44^{\mathrm{a}} \pm 0.016$ & $10.47^{\mathrm{a}} \pm 0.026$ & 10.40 \\
\hline $\mathbf{T}_{\mathbf{6}}$ & $10.25^{\mathrm{b}} \pm 0.017$ & $10.30^{\mathrm{b}} \pm 0.017$ & $10.35^{\mathrm{b}} \pm 0.015$ & $10.40^{\mathrm{a}} \pm 0.023$ & 10.32 \\
\hline $\mathbf{T}_{\mathbf{7}}$ & $10.10^{\mathrm{c}} \pm 0.026$ & $10.12^{\mathrm{bc}} \pm 0.014$ & $10.14^{\mathrm{cd}} \pm 0.023$ & $10.21^{\mathrm{b}} \pm 0.020$ & 10.14 \\
\hline
\end{tabular}

Non-Reducing sugar under ambient condition

The range of non reducing sugar varied from 1.85 to $2.43 \%$. The maximum value for non reducing sugar was $2.43 \%$ recorded for $\mathrm{T}_{7}$
(Oxalic Acid $10 \%$ ) followed by $\mathrm{T}_{1}$ (Chitosan $1 \%)$ i.e. $2.26 \%$. The minimum value for non reducing sugar was $1.85 \%$ recorded for $\mathrm{T}_{0}$ (Control). As compared to $\mathrm{T}_{0}, \mathrm{~T}_{7}$ showed $31.35 \%$ increase in non reducing sugar. 
Table.8 Effect of organic acids on non-reducing sugar (\%) under ambient conditions

\begin{tabular}{|l|l|l|l|l|l|}
\hline \multicolumn{5}{|c|}{ Non Reducing Sugar (\%) } \\
\hline Treatment & Day 1 & Day 3 & Day 5 & Day 7 & Mean \\
\hline $\mathbf{T}_{\mathbf{0}}$ & $1.87^{\mathrm{e}} \pm 0.011$ & $1.86^{\mathrm{e}} \pm 0.014$ & $1.87^{\mathrm{d}} \pm 0.020$ & $1.81^{\mathrm{e}} \pm 0.020$ & 1.85 \\
\hline $\mathbf{T}_{\mathbf{1}}$ & $2.27^{\mathrm{b}} \pm 0.023$ & $2.27^{\mathrm{b}} \pm 0.023$ & $2.25^{\mathrm{b}} \pm 0.017$ & $2.25^{\mathrm{b}} \pm 0.018$ & 2.26 \\
\hline $\mathbf{T}_{\mathbf{2}}$ & $2.14^{\mathrm{c}} \pm 0.022$ & $2.12^{\mathrm{c}} \pm 0.015$ & $2.08^{\mathrm{c}} \pm 0.019$ & $2.06^{\mathrm{d}} \pm 0.023$ & 2.10 \\
\hline $\mathbf{T}_{\mathbf{3}}$ & $2.06^{\mathrm{d}} \pm 0.017$ & $2.05^{\mathrm{d}} \pm 0.020$ & $2.15^{\mathrm{c}} \pm 0.028$ & $2.05^{\mathrm{d}} \pm 0.008$ & 2.07 \\
\hline $\mathbf{T}_{\mathbf{4}}$ & $2.24^{\mathrm{b}} \pm 0.023$ & $2.21^{\mathrm{b}} \pm 0.017$ & $2.07^{\mathrm{c}} \pm 0.052$ & $2.16^{\mathrm{c}} \pm 0.023$ & 2.17 \\
\hline $\mathbf{T}_{\mathbf{5}}$ & $2.06^{\mathrm{d}} \pm 0.020$ & $2.07^{\mathrm{cd}} \pm 0.026$ & $2.07^{\mathrm{c}} \pm 0.023$ & $2.07^{\mathrm{d}} \pm 0.020$ & 2.06 \\
\hline $\mathbf{T}_{\mathbf{6}}$ & $2.15^{\mathrm{c}} \pm 0.024$ & $2.07^{\mathrm{de}} \pm 0.008$ & $2.06^{\mathrm{c}} \pm 0.024$ & $2.05^{\mathrm{d}} \pm 0.008$ & 2.08 \\
\hline $\mathbf{T}_{\mathbf{7}}$ & $2.43^{\mathrm{a}} \pm 0.017$ & $2.44^{\mathrm{a}} \pm 0.017$ & $2.43^{\mathrm{a}} \pm 0.020$ & $2.44^{\mathrm{a}} \pm 0.014$ & 2.43 \\
\hline
\end{tabular}

Total sugar under ambient condition

The range of total sugar varied from 11.65 to $12.59 \%$. The maximum value for total sugar was $12.59 \%$ recorded for $\mathrm{T}_{7}$ (Oxalic Acid
$10 \%$ ) followed by $\mathrm{T}_{5}$ (Citric Acid $10 \%$ ) i.e. $12.46 \%$. The minimum value recorded for total sugar was $11.65 \%$ recorded for $\mathrm{T}_{0}$ (Control). As compared to $\mathrm{T}_{0}, \mathrm{~T}_{7}$ showed $8.07 \%$ increase in total sugar.

Table.9 Effect of organic acids on total sugar (\%) under ambient storage conditions

\begin{tabular}{|l|l|l|l|l|l|}
\hline \multicolumn{5}{c|}{ Total Sugar (\%) } \\
\hline Treatment & Day 1 & Day 3 & Day 5 & Day 7 & Mean \\
\hline $\mathbf{T}_{\mathbf{0}}$ & $11.62^{\mathrm{e}} \pm 0.014$ & $11.62^{\mathrm{e}} \pm 0.011$ & $11.69^{\mathrm{e}} \pm 0.017$ & $11.68^{\mathrm{d}} \pm 0.017$ & 11.65 \\
\hline $\mathbf{T}_{\mathbf{1}}$ & $12.32^{\mathrm{c}} \pm 0.008$ & $12.36^{\mathrm{c}} \pm 0.015$ & $12.43^{\mathrm{b}} \pm 0.020$ & $12.43^{\mathrm{cd}} \pm 0.020$ & 12.38 \\
\hline $\mathbf{T}_{\mathbf{2}}$ & $12.11^{\mathrm{d}} \pm 0.005$ & $12.14^{\mathrm{d}} \pm 0.023$ & $12.18^{\mathrm{d}} \pm 0.018$ & $12.26^{\mathrm{c}} \pm 0.026$ & 12.17 \\
\hline $\mathbf{T}_{\mathbf{3}}$ & $12.17^{\mathrm{c}} \pm 0.011$ & $12.19^{\mathrm{c}} \pm 0.008$ & $12.23^{\mathrm{d}} \pm 0.021$ & $12.32^{\mathrm{b}} \pm 0.017$ & 12.22 \\
\hline $\mathbf{T}_{\mathbf{4}}$ & $12.30^{\mathrm{c}} \pm 0.012$ & $12.29^{\mathrm{b}} \pm 0.011$ & $12.32^{\mathrm{c}} \pm 0.026$ & $12.38^{\mathrm{c}} \pm 0.011$ & 12.32 \\
\hline $\mathbf{T}_{\mathbf{5}}$ & $12.41^{\mathrm{b}} \pm 0.020$ & $12.43^{\mathrm{b}} \pm 0.020$ & $12.47^{\mathrm{b}} \pm 0.025$ & $12.53^{\mathrm{b}} \pm 0.013$ & 12.46 \\
\hline $\mathbf{T}_{\mathbf{6}}$ & $12.38^{\mathrm{b}} \pm 0.014$ & $12.40^{\mathrm{b}} \pm 0.017$ & $12.46^{\mathrm{b}} \pm 0.014$ & $12.44^{\mathrm{c}} \pm 0.014$ & 12.42 \\
\hline $\mathbf{T}_{\mathbf{7}}$ & $12.53^{\mathrm{a}} \pm 0.019$ & $12.57^{\mathrm{a}} \pm 0.023$ & $12.65^{\mathrm{a}} \pm 0.022$ & $12.63^{\mathrm{a}} \pm 0.011$ & 12.59 \\
\hline
\end{tabular}

It is concluded on the basis of above results, it can be concluded that under both storage condition ambient as well as cold. $\mathrm{T}_{1}$ (Chitosan 1\%) treatment showed good results for most of the parameters. The chitosan act as anti-transparent coating for reducing respiration and transpiration rate. Hence, to maintain quality maintenance and shelf life extension of litchi fruit, chitosan may help in formation of novel strategies that could be feasible for storage of litchi fruit on commercial scale. Results of Trial I reveal that $\mathrm{T}_{1}$ (Chitosan $1 \%$ ) was most effective in reducing the rate of weight loss of litchi fruits under ambient conditions.

\section{References}

Ghosh, U., Bhattacharjee, A., Bose, P.K., Choudhuri, D.R. and Gangopadhyay, H., 1998. Physicochemical changes of litchi in modified atmospheric storage.

Huang, P.Y. and Scott, K.J., 1985. Control of rotting and browning of litchi fruit after harvest at ambient temperatures in China. Tropical agriculture, 62(1), 
pp.2-4.

Jiang, Y. and Li, Y., 2001. Effects of chitosan coating on postharvest life and quality of longan fruit. Food Chemistry, 73(2), pp.139-143.

Jiang, Y., Li, J. and Jiang, W., 2005. Effects of chitosan coating on shelf life of cold-stored litchi fruit at ambient temperature. LWT-food Science and Technology, 38(7), pp.757-761.

Ghosh, U., Bhattacharjee, A., Bose, P.K., Choudhuri, D.R. and Gangopadhyay, H., 1998. Physicochemical changes of litchi in modified atmospheric storage.

Jiang, Y.M. and Li, Y.B., 2003. Effects of low-temperature acclimation on browning of litchi fruit in relation to shelf life. The Journal of Horticultural Science and Biotechnology, 78(4), pp.437-440.

Jiang, Y.M., Yao, L., Lichter, A. and Li, J.R., 2003. Postharvest biology and technology of litchi fruit. J Food Agric Environ, 1(2), pp.76-81.

Kumar, A., 2000. Effect of foliar sprays of multi-K on yield quality and shelf life of litchi (Litchi chinensis Sonn.) $c v$. Rose Scented (Doctoral dissertation, Thesis, M. Sc.(Ag.), Horticulture. GB
Pant University of Agric. And Tech., Pantnagar).

Menzel, C. 2001. The physiology of growth and cropping in lychee. South Afr. LitchI Grow.Assoc. Yearbook, 12: 914.

Santerre, C.R., Cash, J.N. and Vannorman, D.J., 1988. Ascorbic acid/citric acid combinations in the processing of frozen apple slices. Journal of Food Science, 53(6), pp.1713-1716.

Sapers, G.M., Hicks, K.B., Phillips, J.G., Garzarella, L., Pondish, D.L., Matulaitis, R.M., McCormack, T.J., Sondey, S.M., Seib, P.A. and Ei-Atawy, Y.S., 1989. Control of enzymatic browning in apple with ascorbic acid derivatives, polyphenol oxidase inhibitors, and complexing agents. Journal of food science, 54(4), pp.997-1002.

Zhang, D. and Quantick, P.C., 1997. Effects of chitosan coating on enzymatic browning and decay during postharvest storage of litchi (Litchi chinensis Sonn.) fruit. Postharvest Biology and Technology, 12(2), pp.195-202.

\section{How to cite this article:}

Ramandeep Kaur, Gurpreet Singh, Deepika Saxena and Gurpreet Singh. 2018. Effect of Post Harvest Treatments for Enhancing Shelf Life of 'Litchi' (Litchi chinensis Sonn.). Int.J.Curr.Microbiol.App.Sci. 7(08): 1040-1047. doi: https://doi.org/10.20546/ijcmas.2018.708.117 